\title{
Prevalência de zumbido, em idosos com e sem história de exposição ao ruído ocupacional
}

\section{Prevalence of tinnitus in elderly individuals with and without history of occupational noise exposure}

\author{
Juliana Jandre Melo', Caroline Luiz Meneses², Luciana Lozza de Moraes Marchiori . \\ 1) Mestre. Coordenadora do Curso de Fonoaudiologia - UNOPAR \\ 2) Mestranda em Ciências da Reabilitação UNOPAR/UEL. Fonoaudiólogia Clínica. \\ 3) Doutora em Medicina e Ciências da Saúde / UEL. Professora do Mestrado em Ciências da Reabilitação da UNOPAR/UEL. \\ Instituição: Universidade Norte do Paraná - UNOPAR \\ Londrina / PR - Brasil. \\ Endereço para correspondência: Campus Universitário de Londrina (Clinica de Fonoaudiologia - UNOPAR) Av. Paris, 675 Londrina / PR - Brasil - CEP: 86041-140 - \\ Caixa Postal: 401 - Telefone: (+55 43) 3371-7775 \\ Artigo recebido em 10 de outubro de 2011. Artigo aprovado em 26 de outubro de 2011.
}

\section{RESUMO}

Introdução: A variedade de alterações metabólicas e circulatórias relacionadas com o ruído pode causar o aparecimento de inúmeros sintomas auditivos, incluindo, zumbido.

Objetivo: O objetivo do estudo foi avaliar a prevalência de queixa de zumbido, em idosos com e sem história de exposição ao ruído ocupacional.

Método: Forma de estudo prospectivo foi realizado em uma amostra populacional com 502 indivíduos com idade superior a 60 anos, através de questionário padronizado e avaliação audiológica. As variáveis estudadas foram frequência de queixa de zumbido e de e história de ruído ocupacional. Foi usada regressão logística para controlar confusão ou modificação de efeito para as outras variáveis sobre as associações de interesse.

Resultado e Discussão: A queixa de zumbido foi verificada em 50\% dos idosos com história de exposição ao ruído ocupacional e em 40,43\% dos idosos sem história de exposição ao ruído ocupacional. Uma alta frequência de queixa de zumbido foi detectada na população sob investigação, porém não houve significativa diferença estatística entre a queixa de zumbido e história de ruído ocupacional.

Conclusão: $O$ resultado desta pesquisa pode ter ocorrido devido à existência de outros fatores que podem ter contribuído para a presença de zumbido, como a própria idade, nos indivíduos sem história de exposição ao ruído ocupacional. Palavras-chave: Zumbido; Ruído Ocupacional; Idoso.

\section{SUMMARY}

Introduction: The various metabolic and circulatory alterations that are related to noise exposure may cause the onset of several symptoms, including tinnitus.

Objective: The purpose of the study was to assess the prevalence of tinnitus complaints in elderly individuals with and without history of occupational noise exposure.

Method: This prospective study was conducted in a sample population consisting of 502 individuals aged over 60 years, by anamnesis and audiological evaluation. The variables that were studied were the frequency of tinnitus and the history of occupational noise. Logistic regression was used to control for potential confusion or modifications caused by the effects of the other variables on the associations of interest.

Results and Discussion: Tinnitus was reported in 50\% of the cases, with tinnitus reported in $40 \%$ of the elderly individuals with history of occupational noise exposure, and in $43 \%$ of controls (elderly individuals without history of occupational noise exposure). A high frequency of tinnitus was detected in the population under investigation, but there were no statistically significant associations between the presence of tinnitus and history of occupational noise exposure.

Conclusion: The results of this study may have occurred due to other factors such as the age of the individuals without history of occupational noise exposure.

Keywords: tinnitus, noise, occupational, aged. 


\section{INTRODUÇÃO}

Indivíduos com Perda Auditiva Induzida por Ruído (PAIR) têm frequentemente vários sintomas, como zumbido, vertigem, diminuição gradual ou distorção no som e alterações na compreensão da fala. O PAIR é irreversível e permanente, mas pode ser prevenida através da utilização de protetores auditivos durante a exposição ao ruído. A magnitude da perda auditiva pode resultar de uma exposição excessiva ao ruído e de fatores associados tais como, nível de pressão sonora, duração, tipo de frequência do ruído, e as características do indivíduo exposto, a susceptibilidade à PAIR, idade, história prévia de alterações auditivas e história familiar de perda auditiva $(1,2,3,4)$.

Diss et al. avaliaram 284 trabalhadores verificando uma prevalência de PAIR de aproximadamente $63 \%$ e zumbido de $48 \%$, encontrando associação entre perda auditiva induzida pelo ruído e zumbido (5).

Já OGido et al, obtiveram relato de zumbidos em $80,81 \%$ da população estudada, concluindo que as disfunções auditivas são queixas frequêntes e que a pesquisa e a avaliação das disfunções auditivas nos exames ocupacionais dos trabalhadores expostos a ruído é fundamental, pois sintomas como zumbidos, podem causar sofrimento e afetar negativamente a qualidade de vida dos trabalhadores (6).

Além de exposições ocupacionais, a perda auditiva, a queixa de zumbido e de vertigem tem sido associadas com o tabagismo, hipertensão, diabetes, envelhecimento, histórico de saúde e atividades de lazer, sendo que a incidência de sintomas no ouvido parece correlacionada coma exposição ao ruído durante a vida inteira (1-3-7,8,9).

A Presbiacusia, que é vista como a terceira condição crônica mais relatada pelos idosos, pode ser definida como a perda auditiva associada ao envelhecimento, refletindo a perda de sensibilidade auditiva associada ao envelhecimento avançado. O típico perfil audiométrico observado clinicamente na presbiacusia é de perda auditiva neurossensorial bilateral simétrica de alta frequência que progride com a idade, sendo que o zumbido surge como um sintoma muito prevalente e de alto impacto na qualidade de vida do paciente senil $(10,11,12)$.

Estudo de FerReira et al, observou que o zumbido é um fator de intensa insatisfação no paciente idoso, por prejudicar suas atividades diárias além de proporcionar alterações emocionais e do sono, correlacionando a presença hipertensão arterial nos pacientes com zumbido embora não tenham encontrado correlação entre o grau audiométrico da perda auditiva e o nível de incômodo sentido pelo paciente (12).

O objetivo do estudo foi avaliar a prevalência de zumbido em idosos com e sem história de exposição ao ruído ocupacional.

\section{MÉTODO}

Um estudo prospectivo foi realizado na UNOPAR em Londrina. O protocolo do estudo foi aprovado pelo comitê de ética da Universidade UNOPAR, com o número $\mathrm{PP} / 0063 / 09$. Esta foi a primeira grande pesquisa rigorosa de examinar 502 idosos na cidade em questão. Os indivíduos foram enviados pelo projeto EELO.

A anamnese incluiu questões sobre idade, sexo, queixa de perda auditiva, zumbido, relacionadas com o trabalho histórico de exposição ao ruído e a história médica. A avaliação audiológica foi realizada individualmente em uma cabine à prova de som com um audiômetro Interactoustics.

A regressão logística foi usada para controlar confusão ou modificação de efeito para as outras variáveis sobre as associações de interesse. Variáveis de confusão que tinham valores de até $20 \%$ de associação com história de ruído ocupacional (modelo univariado) foram inseridas no modelo ajustado de regressão logística. Os procedimentos foram realizados utilizando o software Bio Estat, versão 5.0, adotando um nível de significância de 5\%.

\section{RESULTADOS}

Para o presente estudo foi realizado o cálculo de amostra onde o resultado foi de 382 indivíduos necessários para extrapolar os resultados do estudo à população envolvida na pesquisa.

Considerando possíveis perdas no decorrer da coleta de dados, foram avaliados 519 pacientes, dos quais 502 pacientes foram incluídos nesta pesquisa e 17 pacientes foram excluídos da pesquisa devido à falta em um dos dias de avaliação.

Dos 502 pacientes incluídos no presente estudo, 366 não tinham história de exposição ao ruído ocupacional, representando $72,90 \%$ da amostra, 136 apresentaram histórico de exposição ao ruído ocupacional, representando $27,09 \%$ da amostra.

Dos 502 sujeitos, $286(56,97 \%)$ não apresentaram zumbido e 216 (43,02\%) apresentaram zumbido. Dentre 
os 366 que não foram expostos ao ruído ocupacional, 148 apresentaram zumbido (40,43\%) e 218 não apresentaram zumbido $(59,56 \%)$.

Dos 136 expostos ao ruído ocupacional, 68 (50\%) apresentaram zumbido e 68 (50\%) não apresentaram zumbido (Tabela 1).

Ou seja, 68 sujeitos $(13,54 \%)$ apresentaram zumbido e foram expostos ao ruído ocupacional, 68 sujeitos $(13,54 \%)$ não apresentaram zumbido e foram expostos ao ruído ocupacional, 148 (29,48\%) apresentaram zumbido e não apresentaram ruído ocupacional e 218 (43,42\%) não apresentaram zumbido nem foram expostos ao ruído ocupacional.

Foi realizada estatística através do programa Bio Estat 5.0, utilizando o teste de qui-quadrado, onde se obteve o valor do qui-quadrado de 3,699 e $\mathrm{p}=0,0544$, o que sugere que não existe diferença estatística entre indivíduos expostos ao ruído ocupacional e os não expostos, ao que se refere ao zumbido, quando avaliado sujeitos acima de 60 anos.

Também foi realizado o teste de regressão logística múltipla com 498 sujeitos, onde foram excluídos os pacientes que não haviam realizado alguma das avaliações das variáveis selecionadas: zumbido como variável dependente (y) e exposição ao ruído, idade, gênero, hipertensão e diabetes como variáveis independentes $(\mathrm{x})$.

Observando os resultados pode-se observar que não houve diferença entre as variáveis independentes. Exposição ao ruído $p=0,0580$; idade $p=0,8560$; gênero $\mathrm{p}=0,7544$; hipertensão arterial $\mathrm{p}=0,3006$; diabetes $\mathrm{p}=0,4425$ (Tabela 2).

Tabela I. O zumbido e história de exposição ao ruído ocupacional.

\begin{tabular}{|c|c|c|c|c|c|}
\hline \multicolumn{6}{|c|}{ Ruído Ocupacional } \\
\hline & & SIM & $\%$ & NÃO & $\%$ \\
\hline \multirow[t]{3}{*}{ Zumbido } & SIM & 68 & $13,54 \%$ & 148 & $29,48 \%$ \\
\hline & NÃO & 68 & $13,54 \%$ & 218 & $43,42 \%$ \\
\hline & & 136 & & 366 & \\
\hline
\end{tabular}

\section{DISCUSSÃO}

Dadosdo estudo populacional naárea de gerontológica e geriátrico realizado na Suécia (13) indicam que a deterioração do aparelho auditivo relacionado à idade é mais pronunciada em homens idosos expostos ao ruído comparados com os não expostos ao ruído de 70 anos a idade 75 .

No entanto, um dos fatores limitantes no que se refere ao diagnóstico diferencial das alterações auditivas em idosos é que, normalmente as alterações auditivas relacionada com a idade tendem a ser confundidas pelos efeitos anteriores da exposição ao ruído nos indivíduos empregados anteriormente em um ambiente de trabalho ruidoso (11). No presente trabalho possivelmente a não associação do zumbido com a história de exposição ao ruído pode ter ocorrido devido a esta associação do ruído à idade e a outras comorbidades existentes na população idosa.

Uma vida inteira de exposição ao ruído aumenta as probabilidades de efeitos negativos sobre o aparelho auditivo, mas é difícil determinar a interação entre o ruído, tais alterações e presbiacusia, uma vez que a presbiacusia tem uma etiologia complexa, incluindo tanto fatores intrínsecos e extrínsecos. A influência do ruído sobre a presbiacusia tem sido postulada em numerosos relatórios por quase um século, no entanto, é difícil identificar o ruído prolongado como um único fator para alterações auditivas em idosos (4-14).

As possíveis correlações entre a e história de exposição ao ruído ocupacional em idosos, revela um panorama complexo devido a variedade de fatores associados com a idade. Sendo difícil definir se as alterações auditivas no idoso são causadas apenas pela degeneração associada à idade $(4-9,15)$.

O zumbido, independentemente da queixa auditiva, é um sintoma auditivo muito relatado por indivíduos expostos a níveis de pressão sonora elevados, sendo que vários estudos têm referido que a exposição prolongada do ruído ocupacional pode não somente levar a uma diminuição da audição, mas também ao zumbido e à hiperacusia além de que a exposição excessiva ao ruído é o mais

Tabela 2. Análise de Regressão Logística de zumbido e as variáveis.

\begin{tabular}{lcccc}
\hline VARIÁVEIS & \multicolumn{4}{c}{ REGRESSÃOLOGÍSTICAMÚLTIPLA } \\
Intercepto & Erro padrão & valorp & odds ratio & IC $95 \%$ \\
\hline X I (Exposição ao ruído) & 0,9855 & 0,058 & 1,5168 & 0,99 a 2,33 \\
X2 (idade) & 0,014 & 0,856 & 1,0025 & 0,98 a 1,03 \\
X3 (gênero) & 0,2101 & 0,7544 & 9,364 & 0,62 a 1,41 \\
X4(hipertensão arterial) & 0,1958 & 0,3006 & 1,2247 & 0,83 a 1,80 \\
X5 (diabetes) & 0,2048 & 0,4425 & 1,1703 & 0,78 a 1,75 \\
\hline
\end{tabular}


importante fator de risco para a diminuição da audição e para o aparecimento do zumbido, seguidos da idade e do gênero sendo recomendada a inclusão do tema zumbido em programas de prevenção da perda auditiva a fim de promover a saúde auditiva dos trabalhadores (16).

Dada a alta prevalência de alterações auditivas na população idosa e as diferenças entre os grupos, há uma clara necessidade de compreender sua natureza e causas em vários grupos, a fim de melhorar a prevenção e desenvolver intervenções apropriadas $(4-9,17)$.

\section{CONCLUSÃO}

Este trabalho buscou verificar se a história de exposição ao ruído ocupacional é uma das causas do zumbido no idoso, porém, provavelmente devido a dificuldade na definição das causas das alterações auditivas no idoso, pelas inúmeras comorbidades presentes nessa população e pelo próprio processo de envelhecimento não se verificou significância entre a história de ruído ocupacional e a presença de zumbido nos mesmos. No entanto, a grande frequência de zumbido tanto na população com história de ruído ocupacional como naquela sem o histórico, mostra a necessidade de se estar atento a queixa de zumbido em idosos de modo geral.

\section{REFERÊNCIAS BIBLIOGRÁFICA}

1. Marchiori LLM, Rego Filho E A, Matsuo T. Hypertension as a factor with hearing loss. Rev Bras Otorrinolaringol. 2006, 72(4):533-540.

2. Agrawal Y, Platz EA, Niparko JK. Risk factors for hearing loss in US adults: data from the National Health and Nutrition Examination Survey, 1999 to 2002. Otol Neurotol. 2009, 30(2):139-145.

3. Collee A, Legrand C, Bernadette Govaerts, Van DerVeken P, Boodt F, Degrave E (2011). Occupational exposure to noise and the prevalence of hearing loss in a Belgian military population: A cross-sectional study. Noise \& Health. 2011, 13: (50) 64-70.

4. Marchiori LLM, Melo JJ, Meneses CL. Comparison of hearing loss in Elderly with and without history of occupational noise exposure. Proceeding of the 10th International Congress on Noise as a Public Health, London, 2011.

5. Dias A, Cordeiro R, Corrente JE, Gonçalves CGO.
Associação entre perda auditiva induzida pelo ruído e zumbidos. Cad Saude Publica. 2006, 22(1):63-8.

6. Ogido R, Costa EA, Machado HC. Prevalência de sintomas auditivos e vestibulares em trabalhadores expostos a ruído ocupacional. Rev Saúde Pública. 2009, 43(2): 377-380.

7. Jokitulppo J, Toivonen M, Björk E (2005). Estimated leisure-time noise exposure, thresholds, and hearing symptoms of Finnish Conscripts. Mil Méd. 2006, 171:112116.

8. Marchiori LLM, Rego Filho EA. Queixa de vertigem e hipertensão arterial. Rev. CEFAC. 2007, 9(1):116-121.

9. Poli-Frederico RC, Marchiori LLM, Melo JJ, Carvalho LL. Possible identification of Noise-Induced Hearing Loss susceptibility genes.Proceeding of the 10th International Congress on Noise as a Public Health, London, 2011.

10. Lethbridge-Cejku M, Schiller JS, Bernadel L. Summary health statistics for U.S. adults: National Health Interview Survey. Vital Health Stat. 2004, 10(222).

11. Krishnamurti S. International Journal of Environmental Research and Public Health. 2009, 6(3):889-899.

12. Ferreira LMBM, Ramos Júnior AN, Mendes E P. Caracterização do zumbido em idosos e de possíveis transtornos relacionados. Rev Bras Otorrinolaringol. 2009, 75(2):245-248.

13. Gates AG, Schmid P, Kujawa SG, Nam B, D'Agostino R. Longitudinal threshold changes in older men with audiometric notches. Hear Res. 2000, 141(1-2):220-228.

14. Rosenhall $\mathrm{U}$. The influence of ageing on noise-induced hearing loss. Noise and Health Year. 2003, 5(20):47-53.

15. Mattos LC, Veras RP. The prevalence of hearing loss in an elderly population in Rio de Janeiro: a-sectional study. Rev Bras Otorrinolaringol. 2007, 73(5):654-59.

16. Steinmetz LG, Zeigelboim BS, Lacerda A B, Morata TC, Marques J M. Características do zumbido em trabalhadores expostos a ruído. Rev Bras Otorrinolaringol. 2009, 75(1):714.

17. Pratt SR, Kuller L, Talbott EO, McHugh-Pemu K, Buhari AM, Xu X. Prevalence of Hearing Loss in Black and White Elders: Results of the Cardiovascular Health Study. Journal of Speech, Language, and Hearing Research. 2009, 52: 973 89. 